\title{
A New Recommendation System for Personal Sightseeing Route from Subjective and Objective Evaluation of Tourism Information
}

\author{
Takashi Hasuike *, Hideki Katagiri ${ }^{\dagger}$, \\ Hiroshi Tsuda ${ }^{\ddagger}$
}

\begin{abstract}
This paper proposes a framework to recommend personal sightseeing route as well as to objectively obtain the tourist's utility to sightseeing plans and sightseeing spots from subjective comparison. The subjective comparison is qualitatively performed using a scale of measurement such as Likert scale, and the mathematical programming problem including this comparison as constraints is introduced. In the case of route planning, traveling and sightseeing times are randomly changed dependent on current traffic and congestion conditions, and hence, Time-Expanded Network (TEN) to represent these traffic conditions in the underlying static network with each discrete time step is introduced. In addition, the network optimization problem is introduced to obtain the personal appropriate sightseeing route. This problem is formulated as a nonlinear and discrete optimization problem, and it is hard to solve it directly and efficiently. Therefore, an efficient algorithm is also developed based on dynamic programming and transformation of the main problem into the recursive equation.
\end{abstract}

Keywords: Mathematical programming, sightseeing route recommendation, Time-expanded network, Utility function.

\section{Introduction}

In order to improve both urban and local economics and revitalization, tourism is recently one of the most important topics. Particularly, various research projects of tourism will be started in Japan toward the Olympic and Paralympic games held in Tokyo in 2020. Until now, many ideas for tourism are adopted in many countries and local areas. On the other hand, Information and Communication Technology (ICT) is rapidly developed and widely applied to real world systems. Of course, many tourists can check and refer a lot of tourism information on the Web anytime and anywhere. They can also decide their sightseeing route by themselves using comput-

\footnotetext{
* Waseda University, Tokyo, Japan

† Kanagawa University, Kanagawa, Japan

‡ Doshisha University, Kyoto, Japan
} 
ers and smartphones. Therefore, it is important to design an useful sightseeing route recommendation system according to personal satisfactions.

Previous researches of sightseeing route planning are generally classified into several groups [1]. Particularly, in this paper, A sightseeing route recommendation system from a mathematical point of view is one of important approaches. Particularly, Zhu et al. [15] established a framework of tour planning problem as an integer linear programming problem and developed heuristic algorithms. Abbaspour and Samadzadegan [1] developed a time-dependent methodology of tour planning to design a time-limited tour that collects the maximum total priority value.

However, these previous models based on mathematical models do not include several important factors for the sightseeing route planning. The first is how to set the tourist's priority and satisfaction value for each sightseeing spot objectively. In our previous models [7], we set the satisfaction values as constant numeric values derived from statistical analysis of tourism information. Furthermore, we [6] considered setting the satisfaction values as fuzzy numbers in terms of flexible sightseeing service system and tourists' sensitivities. Fuzzy numbers are based on the fuzzy theory which is one of mathematical approaches to represent the sensitivity numerically. However, it is generally hard to set numerical satisfaction values by the tourist directly. On the other hand, the tourist can compare two sightseeing spots or sightseeing plans such as pairwise comparison according to her/him feelings. For instance, the tourist can evaluate "Sightseeing plan i is better than plan j." and "Sightseeing spot s is far better than spot k." Analytic hierarchy process (AHP) is one of basic methodologies for decision making using the pairwise comparison [3]. Using the AHP, we can obtain the ranking of sightseeing spots and plans according to the tourist's feelings, but cannot obtain her/him utility function for sightseeing. Most recently, Yoshizumi [14] proposed a mathematical programming-based approach to obtain the objective functions from qualitative and subjective comparisons. Using his approach, we can objectively obtain the tourist's utility function for sightseeing to solve a linear programming problem based on the pairwise comparison. In this paper, we apply his interesting approach to personal sightseeing route planning.

The second is how to consider the time-dependent and uncertain factors, particularly traveling and sightseeing times. For instance, the traffic jam tends to happen in the specific time zone such as rush hours and on the main street in the city. Therefore, it is important to collect traffic data and initially estimate the delay time. Thus, we need to integrate time-dependent parameters into the city network and formulate a mathematical model. In mathematical modeling, it is difficult to represent the time-dependent conditions in static network modeling using a standard graph network, because the static network gives only one deterministic value to each edge not depending on clock times. We [5-7] have already published several papers for sightseeing route planning problems with time-dependent parameters by introducing Time-Expanded Network (TEN). A main structure of TEN is to copy the set of nodes in the basic static network for each discrete time step and to connect two nodes with one directed arc according to time-dependency. The advantage of TEN is that it turns the problem of determining an optimal flow over time into a standard network flow problem on the TEN. Therefore, using the advantage, TEN-based models have been proposed for various practical problems $[2,4,11,13]$ as well as our sightseeing route planning problems.

Furthermore, we must consider the real tourists' needs in terms of use-friendly sightseeing service system. In this system, it is generally impossible to set constant satisfaction values, because sightseeing satisfactions are dependent on tourists' sensitivities which are ambiguous. In addition, in Japan National Tourism Organization, several 3-Day model trips are recommended to travel the newly designated theme regions that showcase different aspects of Japanese scenery and culture [10]. Of course, at a specific area such as Tokyo, Kyoto or Hokkaido, it is possible to 
do appropriate planning for the 3-day trip to begin at the accommodation. Therefore, we need to develop a 3-day trip planning system to maximize tourist's satisfaction at a local area based on one specific accommodation. In general, the tourists can do sightseeing more freely on the second day than first and third days, and hence, we develop an appropriate route recommendation system for the second day trip considering tourist's satisfactions. Particularly, we focus on a recommendation ranking system of next sightseeing spots at the current spot based on network optimization and conditional probability derived from statistical and Web data and travel information until current situations. Hasuike et al. [8] proposed a sightseeing route recommendation system considering the above-mentioned conditions. However, the previous model does not consider the rearrangement of the weight of utility function and do recommendation of a new sightseeing spot. Therefore, we improve the previous model to apply various situations in sightseeing.

This paper is organized as follows. In Section 2, we introduce the TEN and explain features of TEN. In Section 3, we introduce constraints derived from TEN and the revised occurrence probability, and formulate our proposed a route recommendation system based on sightseeing route planning problem in network optimization. It is hard to solve our proposed problem directly and efficiently. In real-world application, it is important to require a quick response from our system. Therefore, in this paper, we develop an efficient algorithm based on dynamic programming. Finally, in Section 4, we conclude this paper.

\section{Subjective and Objective Evaluation of Utility Function Based on Mathematical Programming}

An In order to do sightseeing route planning appropriately, it is important to evaluate the tourist's satisfaction for sightseeing objectively. Yoshizumi [14] recently proposed a method to determine an objective function from the experts' evaluations by using a mathematical programming approach. The idea is to use a scale for measuring such as the Likert scale [12] based on paired differences of the objective values, and to formulate these conditions as constraints in mathematical programming.

For instance, we assume that a sightseeing plan has $K$ metrics such as cost, traveling and sightseeing times, mobility, kind of sightseeing spots, and attractiveness and ranking of sightseeing spots and plans set by the decision maker such as tourist companies or evaluation on the Web such as Jalan net [9]. Furthermore, the sightseeing plan is also represented as $K$-dimensional vector $\boldsymbol{y}=\left(y_{1}, y_{2}, \ldots, y_{K}\right)$. As a utility function of the tourist is assumed to be the following weighted sum form in this paper:

$$
f_{w}(y)=\sum_{k=1}^{K} w_{k} y_{k}
$$

where $w_{k},(k=1,2, \ldots, K)$ is the weight of each factor for sightseeing. Using this weighted sum function, if a tourist's evaluations are "Sightseeing plan $i$ is better than plan $j$." and "Sightseeing plan $s$ is far better than plan $k$.", the following inequalities obviously hold:

$$
f_{\boldsymbol{w}}\left(\boldsymbol{y}^{(i)}\right)>f_{\boldsymbol{w}}\left(\boldsymbol{y}^{(j)}\right), f_{w}\left(\boldsymbol{y}^{(s)}\right)>f_{\boldsymbol{w}}\left(\boldsymbol{y}^{(k)}\right)
$$


However, this formulation does not represent the difference between "better than" and "far better than”. Therefore, Yoshizumi [14] introduced the following definition each boundary. $R_{\approx}:$ A tourist evaluates that solution $i$ is "similar to" solution $j$. $R_{>}$: A tourist evaluates that solution $i$ is "better than" solution $j$. $R_{>>}$: A tourist evaluates that solution $i$ is "far better than" solution $j$.

The following situation represents 3 point satisfaction of Likert scale. Of course, it is easy to define 5 or 7 point satisfaction in the same manner. That is, $R_{0}=R_{\approx}, R_{1}=R_{>}$, and $R_{2}=R_{>>}$ in the above-mentioned 3 point satisfaction.

From these definitions, the tourist's pairwise comparison of $n$ alternative sightseeing plans and spots is mathematically represented as the following constraints:

$$
\begin{aligned}
& -b_{0} \leq f_{w}\left(y^{(i)}\right)-f_{w}\left(y^{(j)}\right)+\varepsilon_{i j} \leq b_{0}, \forall(i, j) \in R_{0} \\
& b_{u-1} \leq f_{w}\left(y^{(i)}\right)-f_{w}\left(y^{(j)}\right)+\varepsilon_{i j} \leq b_{u}, \forall(i, j) \in R_{u}
\end{aligned}
$$

where $\varepsilon_{i j}$ is error term derived from the pairwise comparison. Under these constraints, the optimal weight of $f_{w}(y)$ is obtained to solve the following problem [21]:

$$
\begin{aligned}
\underset{w}{\operatorname{Minimize}} & \sum_{i=1}^{n} \sum_{j>i}^{n}\left|\varepsilon_{i j}\right| \\
\text { subject to } & -b_{0} \leq f_{w}\left(\boldsymbol{y}^{(i)}\right)-f_{w}\left(\boldsymbol{y}^{(j)}\right)+\varepsilon_{i j} \leq b_{0}, \forall(i, j) \in R_{0} \\
b_{u-1} \leq & f_{w}\left(\boldsymbol{y}^{(i)}\right)-f_{w}\left(\boldsymbol{y}^{(j)}\right)+\varepsilon_{i j} \leq b_{u}, \forall(i, j) \in R_{u} \\
& \sum_{k=1}^{K} w_{k}=1, w_{k} \geq 0,(k=1,2, \ldots, K)
\end{aligned}
$$

In addition, we can do the equivalent transformation for problem (4) without the loss of optimality by introducing new parameters $\lambda_{i j}^{+}$and $\lambda_{i j}^{-}$as follows:

$$
\begin{array}{ll}
\underset{w}{\operatorname{Minimize}} & \sum_{i=1}^{n} \sum_{j>i}^{n}\left(\lambda_{i j}^{+}+\lambda_{i j}^{-}\right) \\
\text {subject to } & \varepsilon_{i j}=\lambda_{i j}^{+}-\lambda_{i j}^{-}, \lambda_{i j}^{+} \geq 0, \lambda_{i j}^{-} \geq 0, \forall(i, j) \in R_{0} \cup R_{u} \\
& -b_{0} \leq f_{w}\left(y^{(i)}\right)-f_{w}\left(y^{(j)}\right)+\varepsilon_{i j} \leq b_{0}, \forall(i, j) \in R_{0} \\
& b_{u-1} \leq f_{w}\left(y^{(i)}\right)-f_{w}\left(y^{(j)}\right)+\varepsilon_{i j} \leq b_{u}, \forall(i, j) \in R_{u} \\
& \sum_{k=1}^{K} w_{k}=1, w_{k} \geq 0,(k=1,2, \ldots, K)
\end{array}
$$

This problem is a linear programming problem, and hence, it is easy to obtain the optimal weight $w^{*}$ and the corresponding appropriate utility function $f_{w^{*}}(y)$. 


\section{Framework of Personal Route Recommendation System for Sightseeing}

In this section, we propose a mathematical approach to develop a framework of personal route recommendation system for sightseeing on TEN to choice the next sightseeing spot. TEN contains a copy to the set of nodes in the given static traffic network for each discrete time step, and the decision maker can connect two nodes with one directed arc derived from statistical analysis of given traffic data [5]. The advantage of TEN is that it turns an optimal dynamic flow problem over time into a static network flow problem on the TEN which is solved by well-known network optimization approaches.

The main mathematical concept of our proposed system is to recommend the appropriate next sightseeing spot considering the initial route planning in network optimization. First, we assume the following situations.

- The sightseeing route has been recorded as GIS data by the application of Smartphone. Therefore, the decision maker can use the GIS data and current traffic conditions.

-The current sightseeing spot is ranked and evaluated by the tourist using a scale for measuring. This data can be used to revise the tourist's utility function.

-The tourist does not visit each sightseeing spot more than once, i.e., the tourist can visit each sightseeing place only one time.

\subsection{Parameters in Our Proposed Model}

Let $G=(V, E)$ be a connected graph in TEN where $E$ is the set of directed edges and $V$ is the set of nodes. $V$ is also mathematically represented as $M \bigcup\{s, f\}$ where each set is defined as follows:

$s$ : index of departure place

$M$ : index set of sightseeing spots where the tourist can visit. In this paper, we assume $m$ sightseeing places, i.e., $M=\{1,2, \ldots, m\}$

$f$ : index of final destination

The notation of other parameters for the TEN is as follows:

$T$ : target total trip time initially set by the tourist

$t$ : time step on TEN satisfying $t \in S_{t}=\{0, T / N, 2 T / N, \ldots, T\}$ where $N$ is the total step decided by the tourist based on the city transportation network $x_{i j}\left(t, t^{\prime}\right)$ : 0-1 decision variables satisfying the following condition; $x_{i j}\left(t, t^{\prime}\right)=\left\{\begin{array}{l}1, \text { if the tourist travels from spot } i \text { at time } t \text { to spot } j \text { at time } t^{\prime} \\ 0, \text { otherwise }\end{array}\right.$ $x_{i i}\left(t, t^{\prime}\right): 0-1$ decision variables satisfying the following condition; 
$x_{i i}\left(t, t^{\prime}\right)=\left\{\begin{array}{l}1, \text { if the tourist does sightseeing at sightseeing spot } i \text { from time } t \text { to time } t^{\prime} \\ 0, \text { otherwise }\end{array}\right.$

\subsection{Constraints in Our Proposed Model}

TEN-based modeling for sightseeing is generally network optimization. Our proposed model is also formulated as a constrained network optimization problem based on the previous study [5]. The following constraints in the proposed model are derived from general network structures.

(a) Route construction constraint in network optimization

$$
\sum_{t^{\prime} \in S_{t}, t^{\prime}<t} \sum_{i \in M} x_{i j}\left(t^{\prime}, t\right)-\sum_{t^{\prime \prime} \in S_{t}, t<t^{\prime \prime}} \sum_{p \in M} x_{j p}\left(t, t^{\prime \prime}\right)=0,(\forall j \in M, 0<\forall t \leq T)
$$

(b) Departure place constraint

$$
\sum_{t^{\prime} \in S_{t}} \sum_{j \in M} x_{s j}\left(0, t^{\prime}\right)=1
$$

(c) Final destination constraint

$$
\sum_{t \in S_{t}, t^{\prime}<t<T} \sum_{j \in M} x_{j f}\left(t^{\prime}, t\right)=1
$$

(d) Constraint of 0-1 decision variable

$$
x_{i j}\left(t, t^{\prime}\right) \in\{0,1\},\left((i, t),\left(j, t^{\prime}\right)\right) \in A
$$

where $A$ is set of the connected graph derived from obtained TEN with time-dependent parameters $t \leq t^{\prime} \leq T$.

(e) Constraint to each sightseeing place

$$
\sum_{t \in S_{t} t^{\prime} \in S_{t}, t<t^{\prime}} x_{i i}\left(t, t^{\prime}\right) \leq 1, \forall i \in M
$$

\subsection{Formulation of Our Proposed Model}

The objective function in the proposed model is maximizing the tourist's utility given by solving problem (5) from times $t$ to $T$. Therefore, our proposed model is formulated as the 
following TEN-based personal sightseeing route planning problem:

$$
\begin{aligned}
& \text { Maximize } \sum_{i=1}^{m}\left\{f_{\boldsymbol{w}^{*}}\left(\boldsymbol{y}^{(i)}\right)\left(\sum_{t, t^{\prime}} x_{i i}\left(t, t^{\prime}\right)\right)\right\}=\sum_{i=1}^{m} f_{\boldsymbol{w}^{*}}\left(\boldsymbol{y}^{(i)}\right) x_{i} \\
& \text { subject to constraints (a) to (e) }
\end{aligned}
$$

This problem is a simple constrained network optimization problem included in the only original constraint (e) except for standard network structure (a) to (d). The constrained network optimization problem may be solved by using soft computing approaches. However, the main constraints are derived from the standard network structure, and it is important to require a quick response from our system in terms of real-world application. Therefore, we develop an efficient algorithm based on dynamic programming for problem (6).

\subsection{Framework of Our Proposed Sightseeing Route Recommendation System}

The most important factor in our proposed model is to determine the tourist's utility function appropriately. In subsection IV-A to C, the total personal route planning with respect to initial parameter sets is obtained. However, from changes of current traffic conditions and the tourist's utility function for sightseeing, we need to develop the flexible framework of our proposed recommendation system. On the other hand, from the second assumption, the tourist evaluates each spot after sightseeing. In addition, it is not difficult to rearrange another sightseeing route instead of initial route, because the algorithm proposed in subsection IV-C is greedy and efficient. Furthermore, we have obtained and evaluated utilities of each sightseeing spot, and hence, by collecting some features of a new sightseeing spots and clustering the new sightseeing spot among other sightseeing spots, we pick up some new sightseeing spots which include in the group with higher satisfaction value.

Consequently, we propose the following framework to recommend the tourist's sightseeing route by improving Hasuike et al. [8].

\section{Framework of Sightseeing Route Recommendation System}

STEP1: A planner sets $n$ sightseeing plans or spots to do the pairwise comparison by the tourist. In addition, the planner sets numerical evaluations to set $\boldsymbol{y}=\left(y_{1}, y_{2}, \ldots, y_{K}\right)$ in section II. Go to STEP2.

STEP2: The tourist does the pairwise comparison among sightseeing plans, and the planner receives the data as Likert scale point satisfaction. Go to STEP3.

STEP3: The planner solves problem (5) to obtain the tourist's utility function and problem (6) to obtain the optimal sightseeing route. The planner shows the optimal route to the tourist. Go to STEP4. 
STEP4: If the tourist accepts the shown route, the tourist does sightseeing. Go to STEP5. If not, the planner changes the sightseeing plans or spots for the pairwise comparison, and return to STEP 2.

STEP5: During the tourist's sightseeing, the planner receives the current traffic data and the tourist's evaluation data of each spot in the initial route. Go to STEP6.

STEP6: From traffic and evaluation data in STEP5, pick up some new sightseeing spots with the higher satisfaction value using calculated clusters. Go to STEP7

STEP7: The planner resolves problems (4) and (5). If the resolved route is much different from the initial route, the planner tells the revised route to the tourist. If the tourist finishes sightseeing, go to STEP8. If not, return to STEP5.

STEP8: The planner receives the tourist's evaluation of the total plan and revises the recommendation system.

This framework of sightseeing route recommendation system is one of important integrations between ICT and optimization approaches. Each component to obtain the tourist's utility function for sightseeing and optimal route is derived from a simple mathematical programming approach, and hence, our system can be widely applied to real-world tourism.

\section{Numerical Example}

In order to represent to set satisfaction values of sightseeing spots, we provide a numerical example with 6 sightseeing spots and 6 sightseeing plans provided by tourism companies as shown in Table 1 and the tourist does the pairwise comparison as shown in Table 2 using 5 point satisfaction of Likert scale where values 1 to 4 means "fair to”, “a little better than” , "better than" and "far better than", respectively. In addition, value 1/2 means "a litter worse than”. For instance, the tourist evaluates "Sightseeing plan A is far better than plan B.”, and "Plan B is a little worse than plan C.”, that is, "Plan C is a little better than plan B.” We consider that $f_{\boldsymbol{w}}(\boldsymbol{y})=\sum_{k=1}^{6} w_{k} y_{k} \cdot y_{k}$ is $0-1$ variable that $y_{k}=1$ in the case a plan includes sightseeing spot $i$ and $y_{k}=0$ in the case a plan does not include sightseeing spot $i$. In the case that boundary values are $b_{1}=0.025, b_{2}=0.05, b_{3}=0.075$ and $b_{4}=0.1$, the optimal satisfaction values $w_{k}$ are obtained as shown in Table 3 by solving problem (5). 
Table 1. Sightseeing plans provided by tourism companies

\begin{tabular}{|c|c|}
\hline Plan & Sightseeing spots \\
\hline A & $1,2,3$ \\
\hline B & $4,5,6$ \\
\hline C & $1,2,6$ \\
\hline D & $1,3,5$ \\
\hline E & $2,4,5$ \\
\hline F & $3,4,6$ \\
\hline
\end{tabular}

Table 2. Pairwise comparison using 5 point satisfaction

\begin{tabular}{|c|c|c|c|c|c|c|}
\hline & A & B & C & D & E & F \\
\hline A & - & 4 & 2 & 2 & 3 & 3 \\
\hline B & - & - & $1 / 2$ & $1 / 3$ & 1 & 2 \\
\hline C & - & - & - & 1 & 2 & 1 \\
\hline D & - & - & - & - & 3 & 1 \\
\hline E & - & - & - & - & - & $1 / 2$ \\
\hline F & - & - & - & - & - & - \\
\hline
\end{tabular}

Table 3. Optimal satisfaction values of 6 sightseeing spots

\begin{tabular}{|c|c|c|c|c|c|}
\hline Spot 1 & Spot 2 & Spot 3 & Spot 4 & Spot 5 & Spot 6 \\
\hline 0.1202 & 0.2003 & 0.2170 & 0.0868 & 0.1837 & 0.1920 \\
\hline
\end{tabular}

From table 1, spot 4 includes plan B, E and F. From table 2, the tourist considers that plan A not including spot 4 is much better than plan B, E and F. Therefore, the satisfaction value of spot 4 is smallest among all sightseeing spots. Using the optimal satisfactions, the planner can construct the personal sightseeing route.

\section{Conclusion}

In this paper, we have proposed framework of a personal route recommendation system for sightseeing based on mathematical programming techniques to obtain the tourist's utility function and the optimal route. By introducing recent interesting approach to obtain the objective function from qualitative and subjective comparisons, we have show that this idea could be applied to tourism. In addition, in terms of efficiency to obtain the optimal sightseeing route, we have developed a greedy algorithm based on dynamic programming. By solving our proposed problem, the tourist obtains the appropriate sightseeing route quickly. The proposed modeling includes previous useful route planning models and parameter setting approaches for the tourist's satisfaction, and hence, the proposed framework will be more useful.

As an important future work, we need to gather real-world tourism data from smart phones and Web and to analyze the data appropriately using our proposed model. Particularly, it is important to consider what kinds of sightseeing plans the tourism company prepares to obtain the appropriate utility function without unnecessary burden for the pairwise comparison. Furthermore, it is also important to choice appropriate factors $y$. 


\section{References}

[1] R.A. Abbaspour and F. Samadzadegan, "Time-dependent personal tour planning and scheduling in metropolises”, Expert Systems with Applications, 38, pp. 12439-12452, 2011.

[2] F.G. Engineer, G.L. Nemhauser, and M.W.P. Savelsgergh, "Dynamic programming-based column generation on Time-Expanded Network: Application to the Dial-a-Flight problem”, INFORMS Journal on Computing, 23(1), pp. 105-119, 2011.

[3] E.H. Forman and S.I. Gass, "The analytic hierarchy process: An exposition", Operations Research, 49(4), 469-486, 2001.

[4] Y. Guo, T. Mellouli, L. Suhl, and M.P. Thiel, "A partially integrated airline crew scheduling approach with time-dependent crew capacities and multiple home bases", European Journal of Operational Research, 171, pp. 1169-1181, 2006.

[5] T. Hasuike, H. Katagiri, H. Tsubaki, and T. Hiroshi, "A route recommendation system for sightseeing with network optimization and conditional probability", Proceedings of IEEE International Conference on Systems, Man, and Cybernetics (SMC 2015), pp. 2672-2677, 2015.

[6] T. Hasuike, H. Katagiri, H. Tsubaki, and H. Tsuda, "Flexible route planning for sightseeing with fuzzy random and fatigue-dependent satisfactions”, Journal of Advanced Computational Intelligence and Intelligent Informatics, 18(2), pp. 190-196, 2014.

[7] T. Hasuike, H. Katagiri, H. Tsubaki, and H. Tsuda, "Tour planning for sightseeing with time-dependent satisfactions of activities and traveling times”, American Journal of Operations Research, 3(3), pp. 369-379, 2013.

[8] T. Hasuike, H. Katagiri, and H. Tsuda, “A Framework of Route Recommendation System for Sightseeing from Subjective and Objective Evaluation of Tourism Data”, Proceedings of 1st International Conference on Business Management of Technology, pp. 801-806, 2016.

[9] Jalan net (in Japanese), http://www.jalan.net/

[10] Japan National Tourism Organization, http://www.jnto.go.jp/eng/location/routes/rtp/

[11] N. Kliewer, T. Mellouli, and L. Suhl, "A time-space network based exact optimization model for multi-depot bus scheduling”, European Journal of Operational Research, 175, pp. 1616-1627, 2006.

[12] R. Likert, A technique for the measurement of attitudes, Archives of psychology, 1932.

[13] N. Shah, S. Kumar, F. Bastani, and I.L. Yen, "Optimization models for assessing the peak capacity utilization of intelligent transportation systems”, European Journal of Operational Research, 216, pp. 239-251, 2012.

[14] T. Yoshizumi, "A mathematical programming-based approach to determining objective functions from qualitative and subjective comparisons", Proceedings of the Twenty-Ninth AAAI Conference on Artificial Intelligence, pp. 3136-3142, 2015.

[15] C. Zhu, J.Q. Hu, F. Wang, Y. Xu, and R. Cao, “On the tour planning problem”, Annals of Operations Research, 192(1), pp. 67-86, 2012. 